\title{
Remoção cirúrgica de cisto periapical na região anterior da maxila: relato de caso
}

\author{
Surgical removal of periapical cyst in the anterior region of the maxilla: case report \\ Extracción quirúrgica de quiste periapical en la región anterior del maxila: reporte de caso
}

Recebido: 06/11/2021 | Revisado: 1511/2021 | Aceito: 17/11/2021 | Publicado: 24/11/2021

\author{
Mayara Alves de Amorim \\ ORCID: https://orcid.org/0000-0003-0856-222X \\ Faculdade Independente do Nordeste, Brasil \\ E-mail: mayara_alvez@hotmail.com \\ Eduardo Leal Damasceno \\ ORCID: https://orcid.org/0000-0003-2854-7764 \\ Faculdade Independente do Nordeste, Brasil \\ E-mail: lealdudu21@yahoo.com.br \\ Guilherme Guimarães Prates Borges \\ ORCID: https://orcid.org/0000-0002-8424-9529 \\ Faculdade Independente do Nordeste, Brasil \\ E-mail: guilhermegp7@hotmail.com \\ Daniele Figueira Mota de Souza \\ ORCID: https://orcid.org/0000-0002-4859-8326 \\ Faculdade Independente do Nordeste, Brasil \\ E-mail: danielefigueira.52@gmail.com \\ Karina Sarno Paes Alves Dias \\ ORCID: https://orcid.org/0000-0003-4840-2335 \\ Faculdade Independente do Nordeste, Brasil \\ E-mail: karinasarnopa@gmail.com
}

\begin{abstract}
Resumo
Os cistos odontogênicos constituem um grupo de diversas lesões heterógenas. A etiologia do cisto periapical está relacionada ao epitélio do ápice de uma unidade dentária que se encontra com necrose pulpar. A terapia endodôntica convencional é considerada a primeira opção para o tratamento do cisto periapical, no entanto em caso de insucesso, a terapia cirúrgica se faz necessária. O estudo relatar o caso de uma paciente com cisto periapical, realizando uma revisão da literatura sobre o presente temática e enfatizando a importância do exame histopatológico para confirmação do diagnóstico. Trata-se de uma pesquisa descritiva documental, onde serão utilizados os dados coletados em prontuário e fotos da paciente. Paciente do sexo feminino que compareceu a clínica escola de odontologia de uma instituição particular no interior da Bahia encaminhada por um cirurgião dentista para exodontia e remoção de lesão no periápice. A remoção cirúrgica do cisto periapical deve ser levada em consideração quando ocorre insucesso no tratamento convencional endodôntico. É necessário que se faça um exame clínico criterioso e avaliação de exames radiográficos para que seja instituída a melhor terapia, lembrando a importância do exame histopatológico para confirmação do diagnóstico.
\end{abstract}

Palavras-chave: Cisto radicular; Diagnóstico por imagem; Procedimentos cirúrgicos ambulatoriais.

\begin{abstract}
Odontogenic cysts constitute a group of diverse heterogeneous lesions. The etiology of the periapical cyst is related to the epithelium of the apex of a dental unit that has pulp necrosis. Conventional endodontic therapy is considered the first option for the treatment of periapical cysts, however, in case of failure, surgical therapy is necessary. The study reports the case of a patient with a periapical cyst, performing a literature review on the subject and emphasizing the importance of histopathological examination to confirm the diagnosis. This is a descriptive documentary research, where data collected from medical records and photos of the patient will be used. Female patient who attended the dental school clinic of a private institution in the interior of Bahia, referred by a dentist for extraction and removal of a lesion in the periapex. Surgical removal of the periapical cyst should be considered when conventional endodontic treatment fails. It is necessary to carry out a careful clinical examination and evaluation of radiographic exams so that the best therapy can be instituted, bearing in mind the importance of the histopathological exam to confirm the diagnosis.
\end{abstract}

Keywords: Root cyst; Diagnostic imaging; Outpatient surgical procedures.

\section{Resumen}

Los quistes odontogénicos constituyen un grupo de diversas lesiones heterogéneas. La etiología del quiste periapical está relacionada con el epitelio del ápice de una unidad dentaria que presenta necrosis pulpar. La terapia de 
endodoncia convencional se considera la primera opción para el tratamiento de los quistes periapicales, sin embargo, en caso de falla, es necesaria la terapia quirúrgica. El estudio reporta el caso de un paciente con un quiste periapical, realizando una revisión de la literatura sobre el tema y enfatizando la importancia del examen histopatológico para confirmar el diagnóstico. Se trata de una investigación documental descriptiva, donde se utilizarán datos recopilados de historias clínicas y fotografías del paciente. Paciente mujer que acudió a la clínica de la escuela de odontología de una institución privada en el interior de Bahía, remitida por un dentista para extracción y remoción de una lesión en el periapex. Se debe considerar la extirpación quirúrgica del quiste periapical cuando falla el tratamiento endodóntico convencional. Es necesario realizar un examen clínico cuidadoso y una evaluación de los exámenes radiográficos para que se pueda instituir la mejor terapia, teniendo en cuenta la importancia del examen histopatológico para confirmar el diagnóstico.

Palabras clave: Quiste radicular; Diagnóstico por imagen; Procedimientos quirúrgicos ambulatorio.

\section{Introdução}

Os cistos odontogênicos constituem um grupo de diversas lesões heterógenas com características histopatológicas e manifestações clínicas diversas (Cavalcante et al., 2016). De acordo com a Organização Mundial da Saúde (2005),o Cisto Periapical CP de origem inflamatória é o mais comum, sendo conhecido também como cisto radicular.

A etiologia do CP está relacionada ao epitélio do ápice de uma unidade dentária com necrose pulpar, sendo estimulada por uma inflamação (Dexter et al., 2011; Pereira et al., 2013). A lesão periapical é caracterizada por uma cápsula de tecido conjuntivo fibroso decorrente de uma cavidade patológica revestida por epitélio, contendo lúmen e restos celulares chamados de restos epiteliais de Malassez (Neville, 2009; Pereira et al., 2019).

O CP tem por frequência em surgir na porção anterior da maxila e posterior da maxila (Bordini et al., 2013). Esses cistos mostram discreta predileção por homens na quarta e quinta décadas de vida, localizando-se preferencialmente na maxila anterior (Rodrigues, 2017). Nos exames radiográficos, o cisto periapical é evidenciado como uma imagem radiolúcida de densidade homogênea, unilocular e circunscrita, que pode estar associado ao ápice radicular de um ou mais dentes (Oliveira, 2019). Esta lesão não apresenta sintomatologia dolorosa e tem seu crescimento lento, no entanto pode atingir grandes proporções, com presença de tumefação e sensibilidade, além de leve mobilidade (Neville, 2009).

Dentro da literatura são descritas diversas técnicas e tratamento para o CP. De maneira geral a terapia de primeira escolha é convencional clínico-endodôntico podendo ser associado ou não ao ato cirúrgico de remoção ou curetagem para tais lesões. Também, se a lesão apresentar aspecto cístico nos exames imaginológicos, a enucleação total da lesão está indicada (Gomes et al., 2019). A terapia endodôntica convencional é considerada a primeira opção para o tratamento do cisto periapical, no entanto em caso de insucesso, a terapia cirúrgica será necessária. A remoção cirúrgica do cisto periapical dispõe de indicações especificas como risco elevado de fratura do dente durante o acesso ao canal, presença de extenso infiltrado inflamatório na estrutura restante e cistos com grandes dimensões. Nesses casos, o tratamento cirúrgico poderá ser a primeira opção (Oliveira et al., 2018).

Dessa forma, o presente estudo teve como objetivo relatar o caso de uma paciente com cisto periapical, realizando uma revisão da literatura sobre a presente temática e enfatizando a importância do exame histopatológico para confirmação do diagnóstico.

\section{Metodologia}

O presente artigo trata-se de uma pesquisa descritiva documental e foi aprovado pelo comitê de ética em pesquisa da Faculdade Independente do Nordeste, sob o número CAAE: 30442720.8.0000.5578. O mesmo seguiu o protocolo CARE (Consensus-based Clinical Case Reporting) como guia para relatos de casos clínicos (Gagnier et al., 2013). 


\section{Relato de Caso}

Paciente do sexo feminino 48 anos, leucoderma, compareceu a clínica escola de odontologia de uma instituição no interior da Bahia encaminhada por um cirurgião dentista para exodontia do elemento dentário 22 e remoção de lesão no periápice. A mesma tinha como queixa principal aumento no volume em região vestibular do elemento dentário (Figura 1) em questão e relatou já ter realizado ele tratamento endodôntico a cerca de 1 ano.

Figura 1: Imagem do aspecto inicial, tumefação tecidual na região anterior de maxila.

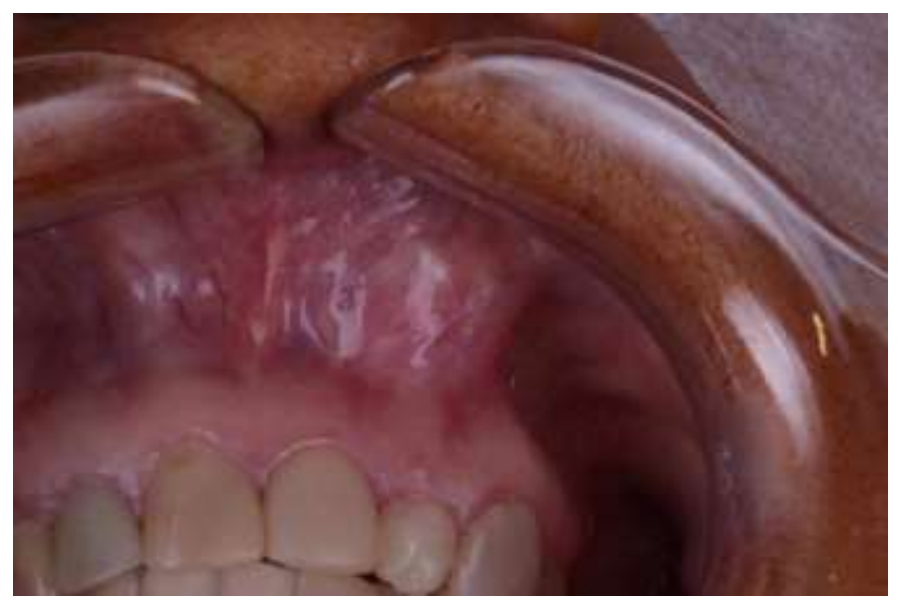

Fonte: Autores.

O acompanhamento radiográfico identificou a não regressão da lesão. Já no exame tomográfico foi observado fratura vertical no elemento dental e presença de lesão de cerca de $2 \mathrm{~cm}$, comprometendo a face vestibular, com destruição de parte do assoalho nasal (Figura 2).

Figura 2: Exame de imagem do tipo tomografia apresentando área hipodensa, bem delimitada, em região de maxila, sugestiva de lesão cística e fratura vertical.

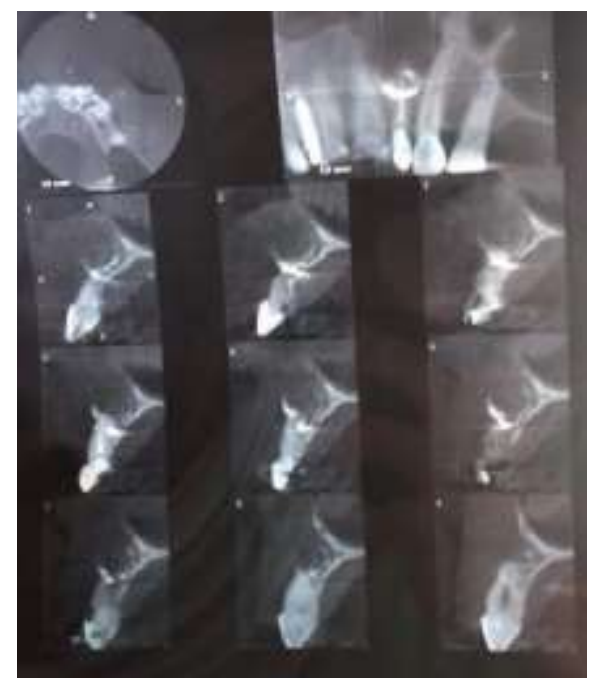

Fonte: Autores.

Dessa forma, foi elaborado o planejamento cirúrgico para exodontia do elemento dentário 22 e remoção total da lesão patológica em região do periápice (Figura 3). 
Figura 3: Exodontia do elemento 22 e remoção total da lesão periapical.

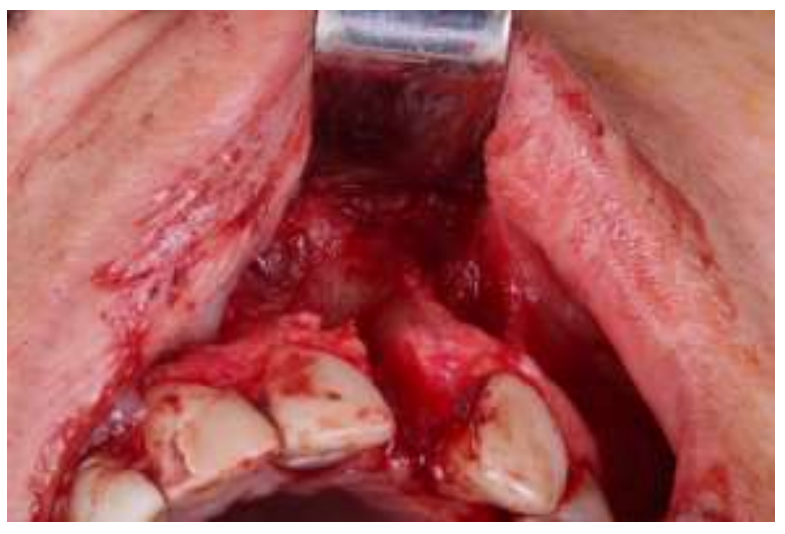

Fonte: Autores.

No primeiro momento foi feita a aferição da pressão arterial da paciente que se apresentou dentro dos padrões de normalidade (120x80mmhg). A antissepsia intra-oral foi feita com digluconato de clorexidina a 0,12\% (PERIOGARD®, São Paulo, Brasil) e extra-oral com Iodopovidona 10\% (RIODEINE®, Rioquímca S/A Ltda., São Paulo, Brasil). A anestesia utilizada foi o bloqueio do nervo alveolar superior anterior e nasopalatino com lidocaína 2\% com epinefrina 1:100:000 (ALPHACAINE®, Nova DFL Ltda., Rio de Janeiro, Brasil) e incisão com cabo de bisturi número 3 (QUINELATO®, Schobell Industrial Ltda., São Paulo, Brasil) e lâmina 15C (LAMEDID@, Bunzl Saúde Ltda., São Paulo, Brasil). O deslocamento do tecido foi realizado com o descolador de Molt nº7 (GOLGRAN MILLENIUM $®$, Golgran Instrumentos Odontológicos Ltda., São Paulo, Brasil) seguido da luxação e remoção do dente 22 com alavancas reta, apical e forcéps nº 150 (GOLGRAN MILLENIUM®, Golgran Instrumentos Odontológicos Ltda., São Paulo, Brasil). Após a remoção foi observada a destruição óssea da parede vestibular. Tendo em vista a lesão, optou-se em realizar a técnica do retalho de Widman modificado para restaurar esteticamente a região (figura 4A). Para as incisões foi utilizado o cabo de bisturi número 3 (QUINELATO®, Schobell Industrial Ltda., São Paulo, Brasil) e lâmina 15C (LAMEDID@, Bunzl Saúde Ltda., São Paulo, Brasil). A primeira incisão foi feita $0,5 \mathrm{~cm}$ apicalmente a margem gengival; a segunda incisão foi intrasulcular na mesma região e a terceira incisão perpendicular e o mais próximo possível da crista óssea do elemento 22, para que se pudesse separar o colar de tecido gengival do osso alveolar com o uso da cureta Mac Call 13-14 (GOLGRAN MILLENIUM®), Golgran Instrumentos Odontológicos Ltda., São Paulo, Brasil). O descolamento mucoperiostal foi realizado com o descolador de Molt 2-4 (GOLGRAN MILLENIUM®, Golgran Instrumentos Odontológicos Ltda., São Paulo, Brasil) e com a lesão patológica exposta pode ser feito um delineamento na região cística com a lâmina 15C (LAMEDID@, Bunzl Saúde Ltda., São Paulo, Brasil) (figura 4B). 
Figura 4: (A) Imagem após o rebatamento do retalho modificado de Widman, onde se pode observar destruição óssea na região vestibular da maxila; (B) Descolamento da lesão; (C) Espécime da lesão após remoção; (D) Comparação ao tamanho da lesão com a lâmina de bisturi.
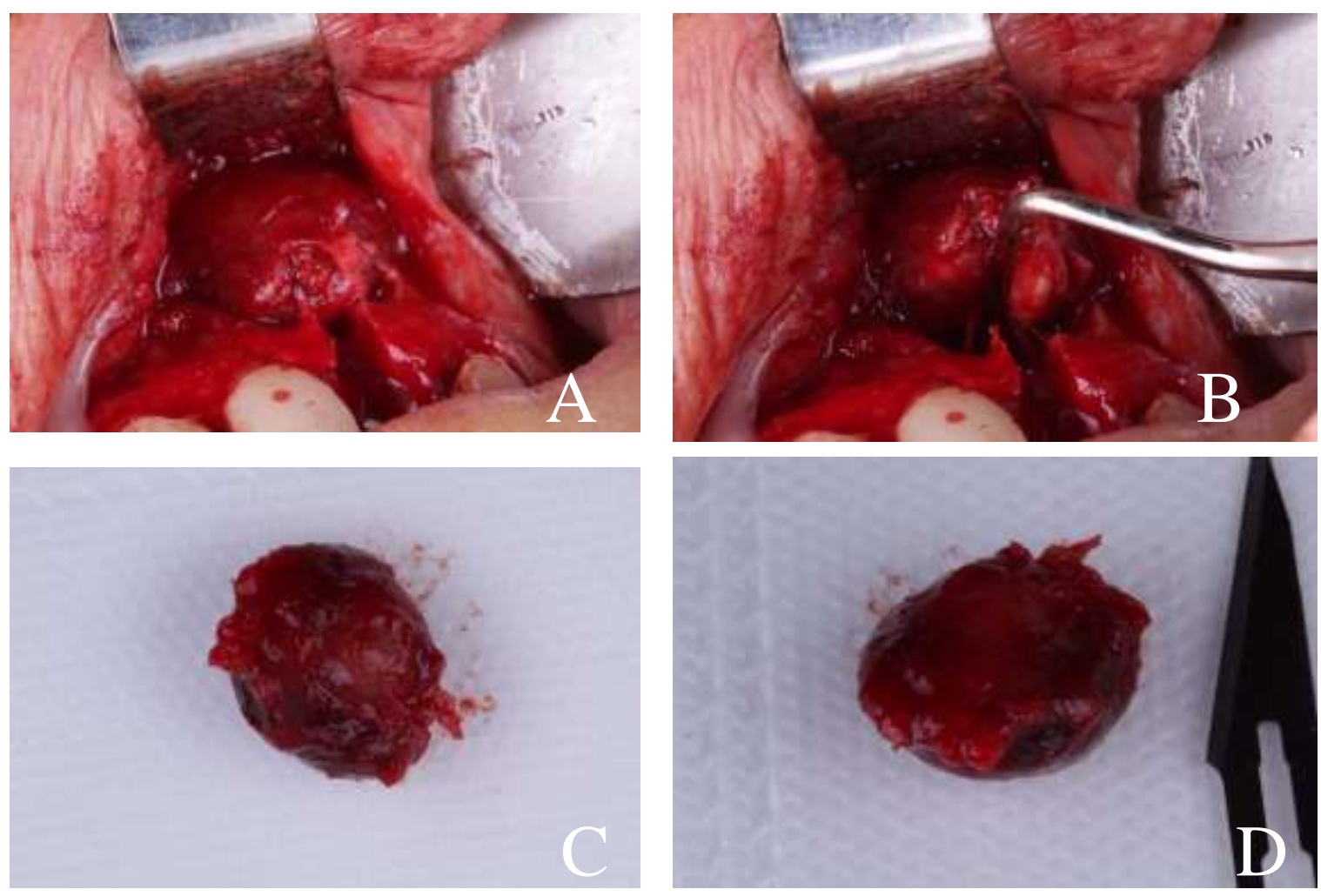

Fonte: Autores.

A lesão cística foi removida com auxílio da pinça hemostática reta (GOLGRAN MILLENIUM®, Golgran Instrumentos Odontológicos Ltda., São Paulo, Brasil), seguida de curetagem do alvéolo com cureta de Lucas (GOLGRAN MILLENIUM®, Golgran Instrumentos Odontológicos Ltda., São Paulo, Brasil) e irrigação com soro fisiológico a $0.9 \%$ (SORIMAX FARMAX®, Farmax Ltda., Minas Gerais, Brasil) para a remoção tecidual que ainda se fazia presente. O procedimento cirúrgico foi finalizado com reposicionamento do retalho e sutura com fio de Nylon 4-0 (PROCARE®, Dental Acess., Rio de Janeiro, Brasil) (Figura 5). 
Figura 5: Aspecto final após a remoção da lesão periapical.

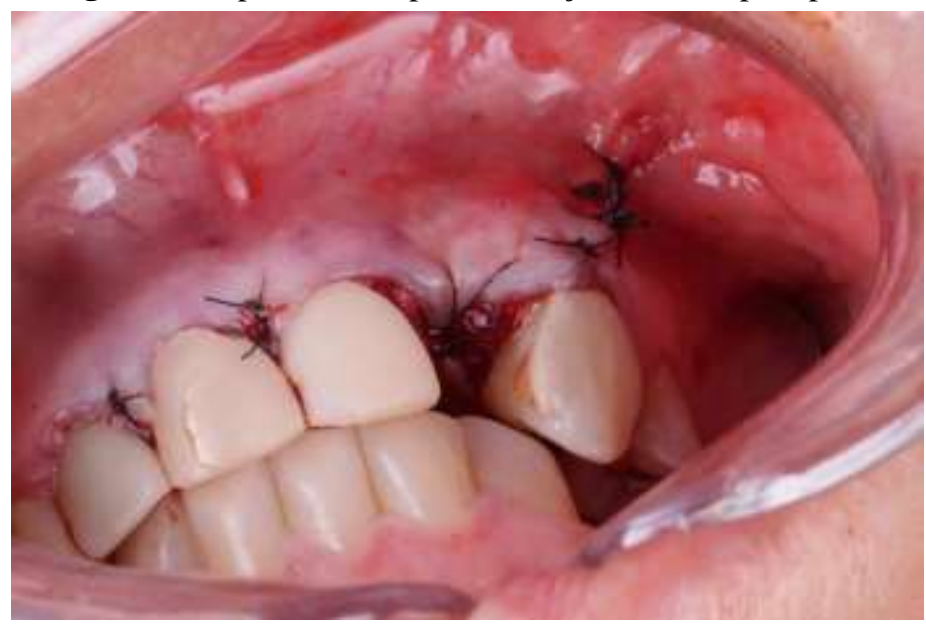

Fonte: Autores.

Não ocorreu complicação transcirúrgica e foi feita a prescrição medicamentosa de Amoxicilina (AMOXIL®, EMS Pharma Ltda., São Paulo, Brasil - 500mg/ 8 em 8 horas/ 7 dias), Dexametasona de 4 mg (DEXAMETASONA Genérico®, EMS Pharma Ltda., São Paulo, Brasil - 4mg/dia/3 dias) e Tylex de 30 mg (TYLEX®, EMS Pharma Ltda., São Paulo, Brasil 30mg/6 em 6 horas/3 dias).

\section{Resultados e Discussão}

O espécime de biópsia incisional foi encaminhado para análise histopatológica e teve o diagnóstico de cisto periapical confirmado (Figura 6). A paciente foi acompanhada por 1 ano após a cirurgia para proservação do caso.

Figura 6: Resultado do exame histopatológico.

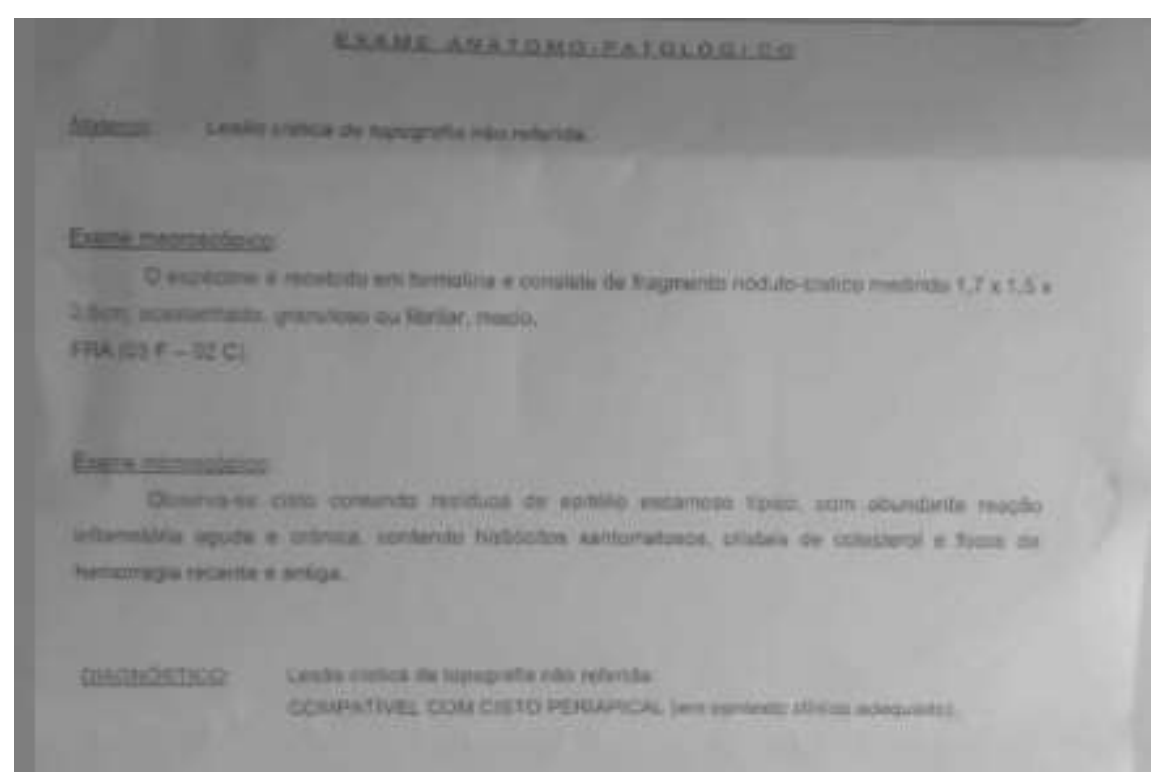

Fonte: Autores.

Cistos periapicais, de origem inflamatória são lesões que se originam a partir de infecções dos canais radiculares provenientes da doença cárie ou traumatismos, que geram alterações pulpares (Silva et al., 2020). Essas alterações são 
originadas por restos epiteliais de Malassez, presentes no ligamento periodontal, que envolvem o dente com aspecto inflamatório periapical crônico (Bilodeau \& Collins, 2017; Resende et al., 2017; Nik et al., 2020).

Normalmente os CPs são resultantes de um processo inflamatório, podendo ser de origem sintomática ou assintomática. Afeta diretamente os tecidos moles e duros das proximidades da raiz dentária, tendo como agente agressor o próprio tecido pulpar necrótico, onde microrganismos se desenvolvem e liberam toxinas (Carneiro et al., 2020). No caso apresentado, o elemento 22, com necrose pulpar, identificada através da radiografia periapical inicial, constituiu-se como o causador da lesão pulpar, sendo, então, indicado o tratamento endodôntico.

Segundo (Netoet al., 2004), o exame radiográfico do cisto periapical não deve ser como determinante para o diagnóstico, pois pode haver outras lesões como o granuloma periapical, que apresentam semelhança radiográfica. A tomografia computadorizada tem sido muito empregada como auxilio em diagnóstico e no planejamento da terapia, visto que auxilia na localização tridimensional da lesão e de estruturas anatômicas adjacentes. (Oliveira et al., 2020). No presente relato, após acompanhamento radiográfico periapical e identificação de não regressão da lesão, além de queixa da paciente de tumefação na região anterior de maxila, foram solicitado exame tomográfico. Observou-se fratura vertical no elemento dentário e uma imagem sugestiva de cisto periapical dada às características: área extensa destruição de cerca de $2 \mathrm{~cm}$, comprometimento da face vestibular e destruição de parte do assoalho nasal. Assim, corroborando com a literatura, o exame tomográfico contribuiu favoravelmente, eliminando as deficiências da visualização bidimensional, apresentadas na radiografia convencional (Holla et al., 2012). No entanto, o diagnóstico da lesão cística só se concluiu após o exame histopatológico, que foi comprobatório para cisto periapical, sendo fundamental para concluir um diagnóstico, descartando outras possíveis lesões (Pinto et al., 2021).

Conforme Laranjeira et al. (2020), descreve quando há insucesso no tratamento endodôntico voltado para as lesões císticas, faz-se necessária a associação com técnicas cirúrgicas. Oliveira et al. (2020); Regezi (2017) relatam que a lesão cística pode ser tratada através da exodontia do elemento dental afetado associada à curetagem da região apical, técnica essa, chamada de enucleação ou marsupialização. No presente caso, essa foi a terapia escolhida, extração do elemento 22 e enucleação com completa remoção do CP.

Estudos apresentam que o acompanhamento para a obtenção do sucesso no tratamento de lesões inflamatórias varia de um a quatro anos (Laranjeira et al., 2020). Neste relato, a paciente está em proservação clínica e radiográfica de um ano em que se observa sucesso após o tratamento realizado.

\section{Conclusão}

A remoção cirúrgica do cisto periapical deve ser levada em consideração quando ocorre insucesso no tratamento convencional endodôntico. É necessário que se faça um exame clínico criterioso e avaliação de exames radiográficos para que seja instituída a melhor terapia, lembrando a importância do exame histopatológico para confirmação do diagnóstico.

\section{Referências}

Bilodeau, E. A., \& Collins, B. M. (2017). Odontogenic cysts and neoplasms. Surgical pathology clinics, 10(1), $177-222$.

Carneiro, M. C., da Costa, F. A., Chicora, P. G. V., Endo, M. S., \& Veltrini, V. C. (2020). Abordagem endodôntica não cirúrgica em extensa lesão periapical: relato de caso. Archives of health investigation, 9(6), 513-516.

Cavalcante, R. M., Costa, M. J. F., Silva, N. S., Souza, L. B., \& Santos, P. P. D. A. (2016). Epithelial odontogenic tumors: analysis of 156 cases in a brazilian population. Int J Odontostomat, 10(1), 113-118.

Da Silva, I. D., Lobo, F., da Silva, B. G., de Souza Tolentino, E., Iwaki, L. C. V., \& da Silva, M. C. (2020). Cistos odontogênicos inflamatórios e de desenvolvimento: estudo observacional e retrospectivo de 23 anos. Revista da Faculdade de Odontologia de Porto Alegre, 61(1), 4-10.

Dexter Brave, M. A., Ramesh, G., \& Brave, V. R. (2011). Radicular cyst of anterior Maxilla. International Journal of Dental Clinics, 3(2), 16-17. 
Gagnier, J. J., Kienle, G., Altman, D. G., Moher, D., Sox, H. \& Riley, D.; Care Group. (2013). The CARE guidelines: consensus-based clinical case reporting guideline development. BMJ Case Rep. 23. 10.1136/bcr-2013-201554.

Gomes, F. V., Gomes, I. A., Gomes, E. V., \& Lara, M. C. R. (2019). Cisto periapical-uma realidade clínico-cirúrgico até os dias atuais: Relato de Dois Casos Clínicos. Revista da AcBO-ISSN 2316-7262, 8(2).

Holla, V. A., Chatra, L., Shenai, P., Rao, P. K., Veena, K. M., \& Prabhu, R. V. (2012). Bilateral inflammatory cysts of the jaw: report of an unusual case. Imaging science in dentistry, 42(2), 105-109.

Laranjeira, L. M. A., Andrade, G. S., de Menezes, A. S. T., da Silva Gaspar, B., de Sousa, Y. R., Mascarenhas, C. L., \& Falcão, I. M. C. (2020). Tratamento de cisto radicular: uma abordagem multidisciplinar. Brazilian Journal of Health Review, 3(3), 6925-6941.

Monteiro, A. S., da Silva, R. L., Oliveira, M. E. D. F. S., Nappi, A. B., Pessuto, A. V. S., Simonato, L. E., \& Fernandes, K. G. C. (2021). Abordagem multidisciplinar em cisto periapical de grande extensão em maxila: relato de caso. Brazilian Journal of Development, 7(9), 90318-90328.

Neto, M. M., Danesi, C. C., \& Unfer, D. T. (2004). Contribuição ao estudo do cisto radicular revisão da literatura. Saúde (Santa Maria), 30(1-2), 90-99.

Neville, B. W., Damm, D. D., Allen, C. M., \& Bouquot, J. E. (2009). Patologia oral e maxilofacial. Rio de Janeiro: Ed. Elsevier, cap, 8, 301-305

Nik Abdul Ghani, N. R., Abdul Hamid, N. F., \& Karobari, M. I. (2020). Tunnel'radicular cyst and its management with root canal treatment and periapical surgery: A case report. Clinical case reports, 8(8), 1387-1391.

Oliveira, k. A., de jesus resende, m. L., \& prado, g. C. P. (2020). Enucleação de cisto periapical em mandíbula: relato de caso. Revista saúde multidisciplinar, 8(2).

Oliveira, L. S. D. S. (2019). Tratamento endodôntico conservador de cisto periapical inflamatório.

Pinto, P. S., de Souza, R. R. L., de Sousa Viana, R., de Morais Neves, L. E., Brito, F. R. C., do Lago, C. A. P., \& Barbosa, L. M. (2021). Abordagem cirúrgica para cisto periapical de tamanho atípico em idosa: Relato de caso. Research, Society and Development, 10 (5), e9210514573-e9210514573.

Pereira, J. A. R., Garcia, K. C., Verbicaro, T., Meger, M. N., de Lucas Corso, P. F. C., \& Scariot, R. (2019). Tratamento cirúrgico de cisto periapical inflamatório de grande extensão em maxila: relato de caso. RSBO, 16(1), 68-76.

Pereira, R. C. (2013). Tratamento de cisto periapical de grande extensão, relato de 2 casos. Monografia de especialização]. Piracicaba: Faculdade de Odontologia de Piracicaba, UNICAMP.

Regezi, J., Sciubba, J. J., \& Jordan, R. C. (2017). Patologia oral: correlações clinicopatológicas. Elsevier Brasil.

Resende, M. A. P., Assis, N. M. S. P., Sette-Dias, A. C., de Aguiar, E. G., \& Sotto-Maior, B. S. (2017). Tratamento cirúrgico e conservador de cisto periapical de grande proporção: relato de caso. HU Revista, 43(2), 191-196.

Rodrigues, J. T., Antunes, H. S., Armada, L., \& Pires, F. R. (2017). Influência da descompressão cirúrgica na expressão de biomarcadores inflamatórios e reparar o tecido em cistos periapicais. Oral Surgery, Oral Medicine, Oral Pathology e radiologia oral; 1-17. 